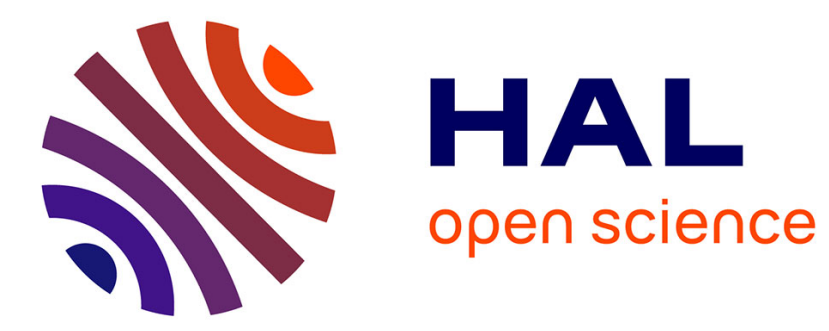

\title{
Emergence and Ubiquity in the Smart Cities
}

\author{
Jose Aguilar
}

\section{To cite this version:}

Jose Aguilar. Emergence and Ubiquity in the Smart Cities. 6th IFIP World Information Technology Forum (WITFOR), Sep 2016, San José, Costa Rica. pp.235-244, 10.1007/978-3-319-44447-5_22 . hal-01429769

\section{HAL Id: hal-01429769 \\ https://hal.inria.fr/hal-01429769}

Submitted on 9 Jan 2017

HAL is a multi-disciplinary open access archive for the deposit and dissemination of scientific research documents, whether they are published or not. The documents may come from teaching and research institutions in France or abroad, or from public or private research centers.
L'archive ouverte pluridisciplinaire HAL, est destinée au dépôt et à la diffusion de documents scientifiques de niveau recherche, publiés ou non, émanant des établissements d'enseignement et de recherche français ou étrangers, des laboratoires publics ou privés. 


\title{
Emergence and Ubiquity in the Smart Cities
}

\author{
Jose Aguilar \\ CEMISID, Departamento de Computación \\ Facultad de Ingeniería, Universidad de los Andes \\ Mérida, Venezuela, 5101 \\ Laboratorio de Robótica y Sistemas Inteligentes \\ Escuela Politécnica Nacional, Quito Ecuador \\ aguilareula.ve
}

\begin{abstract}
This paper aims to add two concepts to the term of Smart City, ubiquity and emergence. The first term used recently in computer science to describe the impact of Information and Communication Technology (ICT) in all areas of society, being the basis of two new research areas that currently have great interest, called the Internet of Things and smart environments. The second term, widely used by some sciences (biology, theology, etc.) to describe the behavior and dynamics that occur in the real phenomena in their areas. In this paper, we analyze their contributions to help to design cities more autonomous, with more capacities of self-management and adaptation.
\end{abstract}

Keywords: Emergent Systems, Ubiquitous Systems, Smart Cities,

\section{Introduction}

The term "smart" is used to define devices such as smart phones, smart cars, smart homes, among others; with different uses and capabilities. The questions to ask are: What makes them smart? Why call them smart? And in the case of this work, What does a city to be "smart"?

In the last twenty years, one of the most innovative industries are aerospace and automotive, based on the introduction of various degrees of automation in its processes. Automation is based in ICT. In fact, some scientists prefer to extend ICTs with the notion of automation (ICAT). The automation are reaching environments common of the life of human beings, with a high level of spatio-temporal integration of technologies with their environments (house, school, etc.).

On the other hand, in the cities there are a lot of "hard technologies" (see [2] for details on that term): telephones, computers, vehicles, among other. There is a long list of technological objects of daily use, which are

adfa, p. 1, 2011.

(C) Springer-Verlag Berlin Heidelberg 2011 
incorporated into our lives. Thus, a city could not stay out of the impact of ICAT, in particular to exploit the information and knowledge that it generates, which can help improve public services, urban planning processes, among others.

For this, it is necessary to analyse how to integrate and exploit the ICAT in the cities, to make it sustainable, to create innovation processes in them, to increase the social, political and economic self-control, etc.

Particularly, the cities should use the ICAT to be smart. They allow computerize, interconnect and automate all processes that occur in a city (energy management, management of traffic, etc.). For a city to become "smart" must perform a transformation process, implementing the ICAT in their core processes. ICAT enables monitor their activities, interconnect the different entities that compose them, transmit information flows, analyse systems that conform them, make smart decisions, etc.

But a first aspect to determine in a city are its essential processes. According to some authors, the main systems in which a city is based are [4], [7], [10], [17]: i) the systems of infrastructure (health, education, etc.). ii) the citizen systems: they define social networking in specific areas of activities in a city (security, civil protection, education, etc.); iii) the production system: includes regulatory frameworks, administrative processes, business networks, etc. iv) the transport system: includes the road network, public transport, etc., v) the communication systems (telephony, Internet, etc.). vi) the water system: covers the supply and sanitation cycle; and the vi) the energy system: includes the generation and distribution infrastructure, and the waste treatment.

These have a huge impact on the dynamics and development of the city. A citizen in a city is continually immersed in these systems, and depend on them to survive [16]. In this context, a city must combine ICAT with their systems, integrating ubiquitously.

This ubiquitous integration of the ICAT in the different components of a city (vehicles, buildings, etc.), become specialized robots that can intelligently respond (for example, a building to the energy system of a city) [11], [15]. This will enable a more sophisticated management of resources, and an emergence of behaviour from the interrelations of these systems, currently unimaginable.

In this context, we can envisage the main systems of a smart city composed by a set of robots (or agents), with autonomous capabilities to work together and to reach collective goals. For example, the vehicles 
coordinate with the smart semaphores and the public transport systems to optimize the traffic; or the buildings and homes work with the energy distribution system of the city to improve the consumption, etc.

Currently there are many initiatives worldwide to improve the capacities of the cities, to meet the demands of the future. Some have called them smart cities, cities of knowledge, among other names. We are interested especially in this work to analyze the term of smart city, in order to extend it with two other terms, indispensable for an autonomous city: emergence and ubiquity, a step beyond the traditional definition of smart city. In this paper, we explore them.

\section{$2 \quad$ Smart Cities}

A smart city uses the ICAT to ensure its energy, environmental, political, economic and social sustainability. In a smart city, the ICAT are integrated into its urban structure, to improve the quality of life or optimize the services provided to citizens.

A smart city incorporates knowledge in the activities of their critical systems. To do this, it requires socializing, researching and analyzing the data, information and knowledge, circulating in the city. The smart city uses them, and incorporates into their decision making processes. Specially, a smart city uses them to improve the functioning of the city and to discover the solutions to its problems. But to succeed, it must study the systems on which it is based, and make them more efficient, more intelligent.

Thus, a smart city is transformed into a "system of systems", using technology [17]. The transformation of a city in "smart" is a complex process. The first step is to develop a transformation plan, where priorities are established, solving several problems at once, since the challenges and threats to sustainability come from all angles and require a holistic strategy.

Many existing smart cities projects focus on specific management solutions and operating services, mainly oriented to energy efficiency. In addition, these solutions do not use ubiquitous computing, and even less semantic mining, which allows interoperability between applications, knowledge discovery, the emergence, etc.

Smart cities should incorporate many technological paradigms to achieve their goals, some of them are [11], [12], [13], [14], [15]: i) the 
Internet of Things paradigm, ii) the cloud computing paradigm, and iii) the big data paradigm.

From the IT point of view, a city can be seen as a concentration of entities (individuals, families, neighborhoods, businesses, schools, public institutions, etc.), through which a lot of information and data flows. In particular, the cities [10], [12]:

- Produce a lot of information and data in different ways.

- Consume much of that information in their internal dynamics.

- Process this information, to be use.

- Store the information, to be used in the time required by the task required.

- Distribute, the information.

A smart city stores all its information. Information is omnipresent in the city, and consumes it in the right place, at the right time. That is, the city makes an intelligent information management, based on the paradigm of ubiquity of information.

\section{$3 \quad$ More than Smart Cities}

Currently there is not a clear definition of smart city, some entities as the IEEE [6] have defined some criteria, but are still very vague. Perhaps for this is that the main objectives of a smart city is to economize (recycle, reuse, etc.) all of their resources (water, energy, waste), but the projects around the smart cities have no clear and concrete proposals about these issues.

Many of the Smart Cities projects focus on concrete solutions of management and operation of services. These solutions do not often use ubiquitous computing, do not allow the emergence of services from the interactions of the entities of the city, and even less semantic information integration that allows the interoperability.

In this section we will introduce two new concepts, Ubiquitous and Emergence. They enable self-adaptation of cities, exploiting the resources available for any task, to make the most sustainable cities.

In the "Ubiquitous and Emergent Cities" the services are not invasive, and the emergence of services as processes of self-organization occur. "Ubiquitous and Emergent Cities" define a new form of interaction among the people with the technology, according to the concept of "calm technology" [9], where the person is the center of the city, and the city is 
imbued with good practice in order to improve the quality of life of its citizens. It implies new human-machine interfaces with forms that allow sensing automatically extract the required information from users to generate responses from the systems.

"Ubiquitous and Emergent Cities" define a new techno-culture, where objects are located both in the urban space and domestic, with a unique identity, to be locatable and traceable, searchable and recyclable. Thus, the "Ubiquitous and Emergent Cities" see all their entities as objects of "internet of things" with:

- Mechanisms to locate them accurately.

- Mechanisms to mine them.

- Tools to handle virtually.

- Mechanisms that allow generate autonomous intelligent behavior.

\subsection{Ubiquitous Cities}

A Ubiquitous City uses the Intelligent environments paradigm to perceive, adapt and respond to people from a perspective in which the information is available anywhere and for whom the require [8], [13], [15]. To do this, the city is seen as a services center, where those services exploit the information available, to provide their capabilities at any time and place (open space and domestic, private and public, etc.).

A Ubiquitous City has a network of services available to citizens from a perspective of prosumers, i.e., citizens with the ability to produce and consume services. That implies certain qualities in the technology of the city, such as [8]:

- Ability to real time processing of large volumes of data.

- Ability to perform tasks of Business Intelligence and Advanced Analytics, permanently and dynamically.

- Based on non-invasive technologies, multi-user systems, multidevice systems, multi-modal systems and multi-platforms systems.

- Based on the paradigm of "context-aware computing".

A ubiquitous city allows the access to all entities that compose it, controlling them remotely, monitoring vital variables (e.g. environmental), among other things, relying on automated systems to aid decision making, prevention systems, among others.

A ubiquitous city needs a middleware based on a service bus, the platform integrates services that enable ubiquity. This makes the 
ubiquitous-as-a-service city in a space of global integration, to catalyze innovation in the city. Thus, the government, citizens, entrepreneurs and researchers are prosumers of services in different areas: education, health, transport, energy, etc. All converge on the platform. Prosumers articulate emerging service environments based on their needs. That makes the ubiquitous city an open ecosystem of integrated management of e-services, which is interactive, intelligent, modular, multimodal, interoperable and adaptive, which allows any person to participate in this new paradigm of social integration. It composes services to govern, to organize business, etc.

Although many of these services already exist today in smart cities, they appear as specific solutions with high proactive participation of users in order to be used. This fragmentation and active participation of users, do not make possible the social "domestication" [5]. Ubiquitous city offers a service architecture across devices "tamed" by society (digital television, smart phones, etc.) to do their daily activities without the user's knowledge. Ubiquitous cities enable the paradigm of einclusion.

In general, services in a ubiquitous city are varied, and are usually based on location (LBS, Location Based Services) such that the information and services provided to users are based on context. The most representative services of a Ubiquitous City are [8], [15]:

- U-Life: These services enable users to control the home equipment automatically. For example, management of climate comfort and energy efficiency at home. Other services in this area are the UEmergency, U-Health, and U-education.

- U-Business: These services involve all areas of the economy, and include U-Banking, U-Payments, U-Work, and U-Logistics.

- U-government: are all the services for the management of local, regional and national governments. Examples are: U-Democracy, UDefense, U-Police, and U-Criminal.

- Intelligent Transport Systems (ITS): these services play a vital role to ensure mobility in the city. Examples are U-logistics, smart cars and smart roads.

- Intelligent Buildings: they are services that enable optimal energy management, security, and the reaction to the context and its users. Examples are U-Office and U-Apartment. 
A wide range of ICAT are necessary in a Ubiquitous City, which are integrated through its middleware. In addition to those already named (Cloud Computing, etc.), it requires other technologies [8]: Computer aware of the context, Augmented Reality, Geographic Information Systems, Global Positioning System (GPS), Broadband Convergence Network (BCN), High Speed Downlink Packet Access (HSDPA) Wireless Broadband (WiBro), Ubiquitous Sensor Network (USN), System-on -a-Chip (SoC), etc.

Some aspects must be considered, to achieve a ubiquitous city [13, [15]:

- It is necessary a computational platform to offer the ubiquity services in the city. Particularly, this services middleware must guarantee integration and interoperability of the services, and must provide security, privacy, among others. With respect to privacy, in [18] is summarized the key challenges and issues, and the emerging technology standards, in the context of privacy and security in smart cities. They establish that the privacy can be achieved: "i) by imposing high security requirements onto the used technology to avoid third party abuses; and ii) by decoupling technical smart city data streams from the personal one to avoid abuse of data by insiders".

- The open data paradigm must be used to allow the utilization of the data in the different contexts.

- It is necessary a synergy between the different actors of the city (government, universities, etc.), to identify the real requirements, to integrate their strengths and to work together, to define the laws for a ubiquitous city, etc.

- It is necessary to promote an innovation ecosystem where emerge the prosumers users, in order to provide unimaginable services, to investigate about the new technologies, their impacts, etc.

\subsection{Emergent Cities}

An U-city should enable the integration of services dynamically. To do so requires ICAT capacities for self-configured according to the needs of citizens. That is, are cities that can self-optimize, self-evolve from its internal dynamics. These are the basis of emergent cities. 
Something will be considered emerging, if we have indicators of the phenomenon on a macro level (observable) [1]. Emergence classic indicators are observable patterns at a higher level, with specific temporal and spatial characteristics. A system emerges in a given environment because it is detectable, and such detection is useful. Emergent systems are based on the logic of the Swarm, communities trying to collectively solve problems that require a lot of flexibility and improvisation. This leads to a Collective, or Social, or swarm, Intelligence in the city, which is reflected in their neighborhoods, in their institutions, and the achievements and goals that arise.

A kind of global wisdom, characteristic of distributed entities interacting, appears in emergent cities, associated with the "Wisdom of Crowds" Paradigm [1]:

- Diversity of Opinion.

- Independence of Opinions

- Aggregation of opinions.

These are the foundations of what some authors have called as Swarmocracia. Moreover, in an emergent city should be given the following characteristics, typical of all emergent system [1]:

- Feedback process.

- Shared memory space.

- Mechanisms and local decision rules.

All this makes the city look like a completely distributed system consisting of heterogeneous entities acting on it. In a city emerges entities, which obey laws of a higher order, which come from that social intelligence. Such entities are able to handle the unpredictability of a city, and consist necessarily of lower-level entities.

The intelligence of an emergent city is based on the combination of telecommunication networks (nerves), the integrated entities ubiquitously (the brains), and sensors and indicators (sensory organs), which operate in the city naturally. It is a web of transport systems, supply energy and water, buildings, household appliances, machinery production, or any management system connections of human activities.

The emergence is because the city produces behaviors in humans prefiguring. These behaviors feed back to the city, so they produce adjustments in it: stores on the boulevards, artists living in specific areas of the city, etc. The city expresses the repeated behavior of groups, collects information on the global behavior, etc. 
An emergent city uses that in its dynamics [11]. For example, an emergent urban planning observes its streets, its operation, the people, etc., and learns from it. From that look, the urban planner tries to extract the identity of the neighborhood, to define the streets, houses, etc. It involves seeing the cities as a machine learning.

Particularly, in an emergent city the citizens must communicate horizontally. Horizontal communication defines forms of dialogue between equals, allowing the emergence of the collective intelligence [1]. A key element is the method to reach collective decisions and avoid conflicts, as part of a process of consultation and dialogue. This method must assume that all options are known by all individuals, and it is necessary to consider all views.

In general, three essential aspects that characterize an emerging city:

- The construction of the shared reality.

- The identity and urban self-reference.

- The ability to self-reproduce.

The self-organizing process of the city, can see in time, as the shops/companies are grouped in very different areas. From a single micro-behavior (close to similar stores), the model is capable of generating a macro-behavior (patterns of organization: location of shops/businesses). For example, it explains the formation of neighborhoods in a city as a unit. These neighborhoods arise from thousands of local interactions that occur in the city. The neighborhoods are patterns over time, emerging from tacit consensus [1]: the financial area is located in a given area, the pubs in other stores, the artisans in another, and so on. The patterns of these urban areas are characterized by their cultural dynamics that determine their activities, people who frequent them, social rules that govern them, urban forms that occur, among other things. The people live in these areas, follow the social norms that are proper to them, without any authority.

Like any emergent system, the city per se is an emergent pattern over time, the product of multiple generations, where the disturbances (changes of government, technological innovations, etc.) are molding it (in Florence, silk spinners have been grouped into the same areas for hundreds of years). Some of these patterns are maintained, because they are linked to physical structures (churches such as San Pedro have allowed the existence of a religious neighborhood around it, etc.), but others have arisen by the laws of emergence (e.g., the neighborhood of silk 
not have a physical structure as attractor) [1]. And this is not because of laziness, it is due to the cultural emergence that occurs on the scale of thousands of years.

\section{Conclusions}

The XXI century will be a century in which cities play a key role in the new global political model. The most successful cities will be those that offer more prosperity to its citizens, overcoming the challenges presented to them for their sustainability. The ICAT are fundamental to it, making them more intelligent, allowing [11]:

- A smart government.

- A digital context to integrate its citizens in the knowledge society.

- A digital social model.

- An electronic economy.

The cities each day will be more embedded in smart devices environments, tools of environmental intelligence and data analytics, among others, in a transparent manner that will individualize services to offer its citizens in the fields of health, culture, among others. These new services have the following characteristics [8], [12], [13], [14], [15]:

- Ubiquity of the user and services.

- Citywide Sensorization

- Adaptation to the user context.

- Semantic technologies.

- Integrated Services

Ubiquitous city allows to see a city as a network of services, available at the appropriate time and place, through the most appropriate device for each situation. The emergent city allows local autonomy, and from there bring out the solutions required for the needs of cities. In that sense, the "Ubiquitous and Emergent Cities" develop an autonomous behavior based on the ICAT, as a result of processes of self-organization. All this allows a Collective Intelligence of a city, with [3]:

- Adaptive, autonomous and intelligent capacities.

- Capabilities to discover and exploit this knowledge.

- Capacity to integrate and automate social processes.

- Capacities to exploit the components deployed in the city. 
But, the social intelligence emerges in a city, if there is:

- An empowerment of the intelligence.

- An intelligent instrumentation.

- The orchestration of efforts.

Particularly, an essential element of a ubiquitous and emergent city are spaces to build social consensus. These spaces are an essential element of life in the city. In these spaces are given dynamics of recognition of diversity, such that different views can be discussed and explored. It is not from the imposition but from the dialogue as a way of building social agreements, like a "ubiquitous and emergent city" defines its future. These consensuses make the development proposals emerge from the local level, which makes possible the emergence of a new citizen, responsible, constructive, among other qualities. Emergent systems provide concrete ways to do this.

In this context, it is important to define a Collective/Social Intelligence. This type of intelligence is based on the recognition of the diversity (social, political, etc.) of the citizens, in the respect to the others (including the earth), in the definition of common public benefits, etc. The social intelligence is reached when the communities are capable to agree, to build collective agreements, in order to achieve a good living. In this case, a "smart, ubiquitous and emergent" city, will simply follow these social patterns.

\section{Acknowledgements}

Dr Aguilar has been partially supported by the Prometeo Project of the Ministry of Higher Education, Science, Technology and Innovation of the Republic of Ecuador.

\section{References}

1. Aguilar, J., Introducción a los Sistemas Emergentes, Mérida, Venezuela, Talleres Gráficos, Universidad de Los Andes (2014).

2. Aguilar, J., Hacia una Tecnología Democrática para Mérida: bases para un nuevo Paradigma Universitario en la creación de una Facultad en Tecnologías Informáticas, Revista de la Academia de Mérida, 14, 17-80 (2009).

3. Aguilar, J., Besembel, I., Cerrada, M. Hidrobo, F., Narciso, F. Una metodología para el Modelado de Sistemas de Ingeniería Orientado a Agentes, Revista Iberoamericana de Inteligencia Artificial, 12, 39-60 (2008). 
4. Batty M., Axhausen K., Fosca G., Pozdnoukhov A., Bazzani A., Wachowicz M., Ouzounis G., Portugali Y. Smart cities of the future, The European Physical Journal Special Topics, 214, 481-518 (2012).

5. Clohessy T., Acton T., Morgan L. Smart City-as-a-Service (SCaaS): A Future Roadmap for E-Government Smart City Cloud Computing Initiatives. IEEE/ACM 7th International Conference on Utility and Cloud Computing, Londres, UK, 836-841 (2014).

6. IEEE smartcities. In http://smartcities.ieee.org/ (2013).

7. Komninos, N. What makes cities intelligent?. Smart Cities: Governing, Modelling and Analysing the Transition (D. Mark Ed.). Taylor and Francis. 77-87 (2013).

8. Lee, S., Han, J., Leem, Y., Yigitcanlar, T. Towards ubiquitous city: Concept, planning, and experiences in the Republic of Korea. Knowledge-Based Urban Development: Planning and Applications in the Information Hershey (T. Yigitcanlar, K. Velibeyoglu \& S. Baum Eds.), PA, IGI Global Era, 148--169 (2008).

9. Lombardi, P., Giordano, S., Farouh, H. Yousef W. Modelling the smart city performance, 2, 137-149 (2012).

10. Taewoo, N. Pardo, T. Conceptualizing smart city with dimensions of technology, people, and institutions. 12th Annual International Digital Government Research Conference: Digital Government Innovation in Challenging Times, New York, NY, USA, 282-291 (2011).

11. Bezerra R., Nascimento F., Martins J., On computational infraestruture requirements to smart and autonomic cities framework, IEEE First International Smart Cities Conference (ISC2), Guadalajara, 1-6 (2015).

12. Jaloudi, S. Open source software of smart city protocols current status and challenges, International Conference on Open Source Software Computing (OSSCOM), Amman, 1-6 (2015).

13. Khan Z., Pervez Z., Ghafoor A., Towards Cloud Based Smart Cities Data Security and Privacy Management, IEEE/ACM 7th International Conference on Utility and Cloud Computing (UCC), London, 806-811 (2014).

14. Leon-Garcia A., Tizghadam A., Application platforms for smart cities, IEEE First International Smart Cities Conference (ISC2), Guadalajara, 1-6 (2015).

15. Nandury S., Begum B., Smart WSN-based ubiquitous architecture for smart cities, International Conference on Advances in Computing, Communications and Informatics (ICACCI), Kochi, 2366-2373 (2015).

16. Oliveira A., Campolargo M., From Smart Cities to Human Smart Cities, 48th Hawaii International Conference on System Sciences (HICSS), Kauai, HI, 2336-2344 (2015).

17. Peltan T., Smart Cities as complexity management, Smart Cities Symposium Prague (SCSP), Prague, 1-5 (2015).

18. Sen M., Dutt A., Agarwal S., Nath A., Issues of Privacy and Security in the Role of Software in Smart Cities, International Conference on Communication Systems and Network Technologies (CSNT), Gwalior, 518-523 (2013). 